\title{
Tuberculosis cutánea a forma de lupus vulgar causada por vacuna de bacilo Calmette-Guérin. A propósito de un caso pediátrico
}

\author{
María Belén Amorín Casella*, Cecilia Coitinho†
}

\section{Resumen}

La tuberculosis cutánea es una enfermedad infecciosa crónica y poco frecuente ocasionada por Mycobacteriun tuberculosis. Existen varias formas de la infección cutánea, siendo el lupus vulgar la más frecuente y común en los países industrializados. Las variantes clínicas dependerán de la vía de llegada del bacilo a la piel, del estado inmunológico del paciente y del medio ambiente. En el lupus vulgar, además de M. tuberculosis puede estar implicado M. bovis y menos frecuentemente el bacilo de Calmette y Guérin. Esta entidad presenta una evolución crónica, es de carácter progresivo y es rara de ver en niños.

Se presenta el caso de una niña en la cual se diagnosticó una forma de tuberculosis cutánea denominada lupus vulgar provocada por el bacilo de Calmette y Guérin.

Caso clínico: niña de 10 años que presentaba lesiones cutáneas de diferentes características, de evolución tórpida y asintomáticas; la de mayor tamaño se localizaba en la cara externa del deltoides derecho en forma de placa eritematosa de 20 por $10 \mathrm{~cm}$ con bordes sobreelevados presentando en su centro micropápulas que coalescen dejando una zona de atrofia central. Esta había comenzado en etapa neonatal luego de la vacunación por el bacilo de Calmette y Guérin. Desde hacía tres años presentaba una segunda lesión más pequeña en cara externa de muslo derecho de similares características y otras de carácter múltiple en dorso con forma de micropápulas rojo vinosas. Los exámenes de valoración general fueron normales, al igual que la radiografía de tórax y de los miembros. El test de la tuberculina mostró una induración de $15 \mathrm{~mm}$ y las baciloscopias fueron negativas. La biopsia de la lesión de piel del deltoides mostró granulomas caseosos y las técnicas moleculares diagnosticaron Mycobacterium bovis atenuado. Con ese diagnóstico, recibió tratamiento antituberculoso por diez meses con buena tolerancia y con mejoría de las lesiones.

Palabras clave: Tuberculosis cutánea Mycobacterium tuberculosis Vacuna BCG Lupus vulgar Mycobacterium bovis Niño
Key words: $\quad$ Tuberculosis, cutaneous Mycobacterium tuberculosis BCG vaccine Lupus vulgaris Mycobacterium bovis Child

\footnotetext{
* Prof. Adj. Pediatría, Paysandú. Diplomada en Infectología Pediátrica.

† Médico del Departamento de Laboratorio de la Comisión Honoraria para la Lucha Antituberculosa y Enfermedades Prevalentes. Centro de Referencia Nacional para Micobacterias. Ministerio de Salud, Uruguay.

Comepa, Paysandú.

Correspondencia: María Belén Amorín Casella. 33 orientales 937/1002, Paysandú. Correo electrónico: belen@paysandu.com Artículo con consentimiento informado de publicación de parte de los responsables del menor.

Recibido: 28/11/18

Aprobado: $11 / 3 / 19$
} 


\section{Introducción}

La tuberculosis constituye hoy día una pandemia mundial. En el 2016 se registraron 10,4 millones de nuevos casos con una tasa de incidencia anual estimada de 125 cada 100.000 habitantes que varía según el país. Es la novena causa de muerte y la primera por un agente infeccioso, ocasionando más de 1.500 .000 muertes anuales. Existe un aumento de las formas graves y extrapulmonares, relacionadas con la infección por el virus de la inmunodeficiencia humana (VIH), aumento de personas institucionalizadas y deterioro de las condiciones socioeconómicas de la población ${ }^{(1,2)}$.

Nuestro país no escapa a este problema. Actualmente ocurren 29 casos por 100.000 habitantes siendo las formas pleuropulmonares las más frecuentes, mientras que las extrapulmonares se registran en menor proporción. Desconocemos qué sucede con las formas cutáneas. Sabemos que a nivel mundial estas representan entre $1 \%$ y $4 \%$ de las formas extrapulmonares, porcentaje que varía según el área geográfica ${ }^{(3-6)}$. No existen publicaciones respecto al tema y referencias sobre las complicaciones por bacilo de Calmette y Guérin (BCG).

La tuberculosis cutánea se produce por inoculación directa del bacilo en la piel o por vía endógena, ya sea por diseminación hematógena o linfática desde un foco contiguo en un paciente sensibilizado con alto grado de inmunidad a M. tuberculosis ${ }^{(7)}$. En el caso de la inoculación exógena el ingreso al tejido cutáneo se realiza a través de abrasiones, colocación de pendientes, inyecciones con jeringas no esterilizadas, circuncisiones rituales, tatuajes y vacunación con BCG.

Dentro de las formas de tuberculosis cutánea, el lupus vulgar (LV) constituye la forma más frecuente y conocida en países industrializados. Su principal agente causal es M. tuberculosis, pero también puede estar implicado M. bovis y menos frecuentemente BCG. Cuando es producida por la cepa vacunal el riesgo se estima en 5 por 1.000 .000 o 1 en 100.000 casos de vacunados ${ }^{(8)}$. El BCG es una cepa atenuada de $M$. bovis que se usa en la vacunación rutinaria de los recién nacidos inmunocompetentes en países con altas tasas de tuberculosis, incluido el nuestro. Su eficacia ha sido documentada en prevenir las formas graves de la enfermedad ${ }^{(9,10)}$. Es una vacuna relativamente segura siendo las complicaciones muy raras de observar, a excepción de niños con inmunodeficiencias primarias (IDP) o secundarias.

La importancia del LV radica en su perfil crónico y progresivo. Se caracteriza por una placa eritematosa con múltiples nódulos en jalea de manzana que se extienden irregularmente con formación de cicatriz y destrucción del tejido. Es una infección que librada a su evolución natural puede provocar severas cicatrices, deformidades del miembro y transformarse con los años en un carcinoma escamoso ${ }^{(2,7)}$. El diagnóstico no es fácil dados los múltiples diagnósticos diferenciales y la ausencia de bacilos en las lesiones. En ese caso se hace necesario realizar una biopsia de las lesiones y la búsqueda de bacilos ácido alcohol resistentes (BAAR) mediante cultivo o técnicas moleculares.

Se comunicará el primer caso pediátrico en nuestro país de una enfermedad poco frecuente con las dificultades diagnósticas que determinaron su larga evolución y se realiza además una revisión de las diferentes cepas vacunales del BCG y sus complicaciones.

\section{Caso clínico}

Niña de 10 años, procedente de Paysandú, de padres no consanguíneos y de medio socioeconómico deficitario. Buen crecimiento y desarrollo, portadora de un asma leve. Como antecedente familiar se destaca que un tío paterno y el padre de la niña presentaron una lesión en axila derecha luego de la vacuna por el BCG, por lo cual el padre recibió tratamiento antituberculoso en la niñez, con curación de la lesión.

Consulta en la policlínica de infectología debido a lesiones de piel de varios años de evolución y con el resultado de una biopsia cutánea que informaba una dermatitis nodular granulomatosa de tipo necrotizante compatible con tuberculosis cutánea sin visualización de BAAR con las técnicas utilizadas.

La lesión de mayor entidad había comenzado luego del nacimiento, tras la administración de la BCG. A los pocos días de vida los padres relataban la formación de una tumoración en axila derecha que posteriormente se abscedó y que luego de semanas curó espontáneamente. Luego notan la presencia de una placa eritematosa ubicada en la cara externa del deltoides derecho que deja áreas cicatrizales sobre las cuales reaparecen nuevas lesiones, de evolución tórpida que no mejora a pesar de recibir múltiples tratamientos tópicos. En los últimos tres años agrega una lesión similar en muslo derecho. No presentaba dolor, pero a veces relataba un leve prurito. $\mathrm{Al}$ examen físico se destacaba un buen estado general, afebril, en la piel se observaba una lesión en cara externa del deltoides derecho tipo placa indolora, eritematosa, de 20 por $10 \mathrm{~cm}$ con bordes sobreelevados y descamaste donde se observan micropápulas que coalescían dejando una zona de atrofia central. Presentaba otra lesión similar pero más pequeña, de 5 por $5 \mathrm{~cm}$, eritematosa y más redondeada en cara externa de muslo derecho. En axila se observaba una cicatriz pequeña de tipo queloide (figura 1). En dorso se observaban otras lesiones, hipopigmentadas y micronodulares de color rojo vinoso de 2 por $2 \mathrm{~cm}$ (figura 2). 


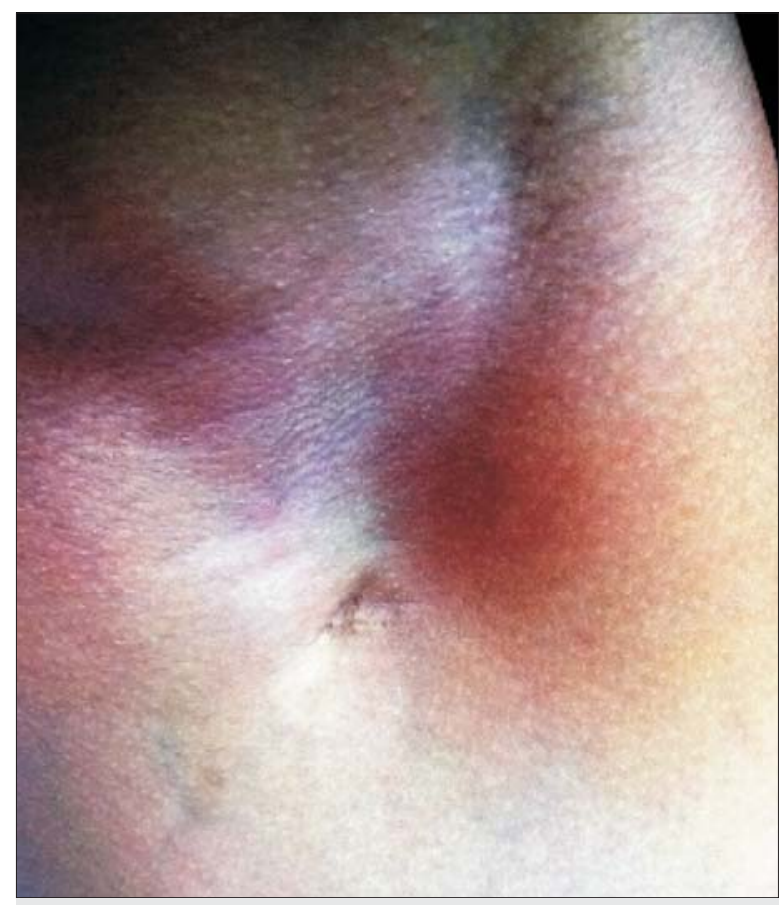

Figura 1. Lesión cicatrizal deprimida en axila derecha de la lesión referida en etapa neonatal.

Dados los resultados de la biopsia de piel que informaba una tuberculosis cutánea se solicitó el test derivado de la proteína purificada (PPD) o tuberculina que reveló una induración de $15 \mathrm{~mm}$, las baciloscopias fueron negativas y las radiografías de tórax y de miembros superior e inferior derechos no mostraron alteraciones. El hemograma, funcional hepático, velocidad de eritrosedimentación, proteína $\mathrm{C}$ reactiva e inmunoglobulinas fueron normales. $\mathrm{La}$ ecografía abdominal no mostró alteraciones y el VIH y VDRL resultaron no reactivos. Se solicitaron poblaciones linfocitarias que no se realizaron.

Debido a que no se contaba con la etiología y era fundamental para iniciar el tratamiento, se solicitó una nueva biopsia cutánea, que se consiguió luego de ocho meses debido a la renuencia de los padres a repetir el procedimiento.

La segunda muestra informó un proceso inflamatorio granulomatoso en la dermis con el detalle de un granuloma con la corona linfocitaria y células gigantes multinucleadas (figura 3). En esa oportunidad, previa coordinación, se envió al Laboratorio de la Comisión Honoraria de la Lucha Antituberculosa (LAB-CHLA), donde se identifica Mycobacterium tuberculosis complex (MTBC), que mediante hibridación por sondas (Genotype MTBC) se identifica la subespecie Micobacterium bovis atenuado (BCG). Tanto el M. bovis como el BCG son intrínsecamente resistentes a la pirazinamida, por lo cual no se determinó la sensibilidad. La niña recibe isoniacida, etambutol y rifampicina diaria de lunes a sábado por cinco meses y luego trisemanal solo con isoniacida y rifampicina completando diez meses de tratamiento. No presentó efectos adversos a los fármacos y en el último control habían desaparecido las lesiones del dorso y se observó una mejoría de las lesiones de los miembros (figura 4).

\section{Discusión}

La tuberculosis cutánea es una enfermedad infecciosa de evolución crónica, poco frecuente y de difícil diagnóstico. Suele ser confundida con múltiples patologías que comprometen la piel como son las micosis profundas, leishmaniasis cutánea o cáncer. La piel es un órgano poco receptivo para el bacilo de Koch, lo que provoca manifestaciones diversas y con escasos bacilos (formas paucibacilares). Desconocemos qué diagnósticos diferenciales se realizaron en la paciente previo a esta consulta, pero fue tratada con múltiples cremas y ungüentos con y sin antibióticos durante años sin mejoría. Luego de realizada la biopsia se confirma la característica paucibacilar de las lesiones al no mostrar los bacilos.

La infección puede ser endógena a partir de una lesión tuberculosa que se origina por contigüidad de un foco subyacente (óseo, ganglios, vainas tendinosas) o puede adquirirse por vía linfática o diseminación hematóge$\mathrm{na}^{(4,5)}$.

En el caso de infección exógena, situación menos frecuente, los bacilos invaden la piel desde el exterior o puede también darse el caso que el huésped haya estado en contacto con el M. tuberculosis generando una tuberculosis de reinfección. La inoculación exógena puede dar como resultado un chancro de inoculación primario (primoinfección), una tuberculosis cutánea verrucosa cutis o un LV, dependiendo de la ausencia o presencia de hipersensibilidad del huésped.

Existen varias clasificaciones de la tuberculosis cutánea. La más aceptada ha sido la de Beyt y colaboradores, que tiene en cuenta la vía de la infección. Sin embargo, han surgido otras, como la de Tappeiner y colaboradores, que consideran la de Beyt pero la amplían, incluyendo la forma por BCG y las tuberculides. La clasificación actual es la de Sehgal y colaboradores que enfatizan en tres factores: el agente infeccioso, la inmunidad del huésped y la ruta de la infección. De acuerdo con esta interpretación, el LV, la tuberculosis verrucosa cutis y el chancro de inoculación se comportarían como una reinfección, donde hay una historia de infección previa o vacunación como sucedió en este caso. En el otro extremo estarían las formas de reactivación como la escrófula, la tuberculosis orificial y la tuberculosis miliar ${ }^{(1,4,10)}$.

El LV constituye la forma más frecuente y conocida de tuberculosis cutánea en países industrializados. 


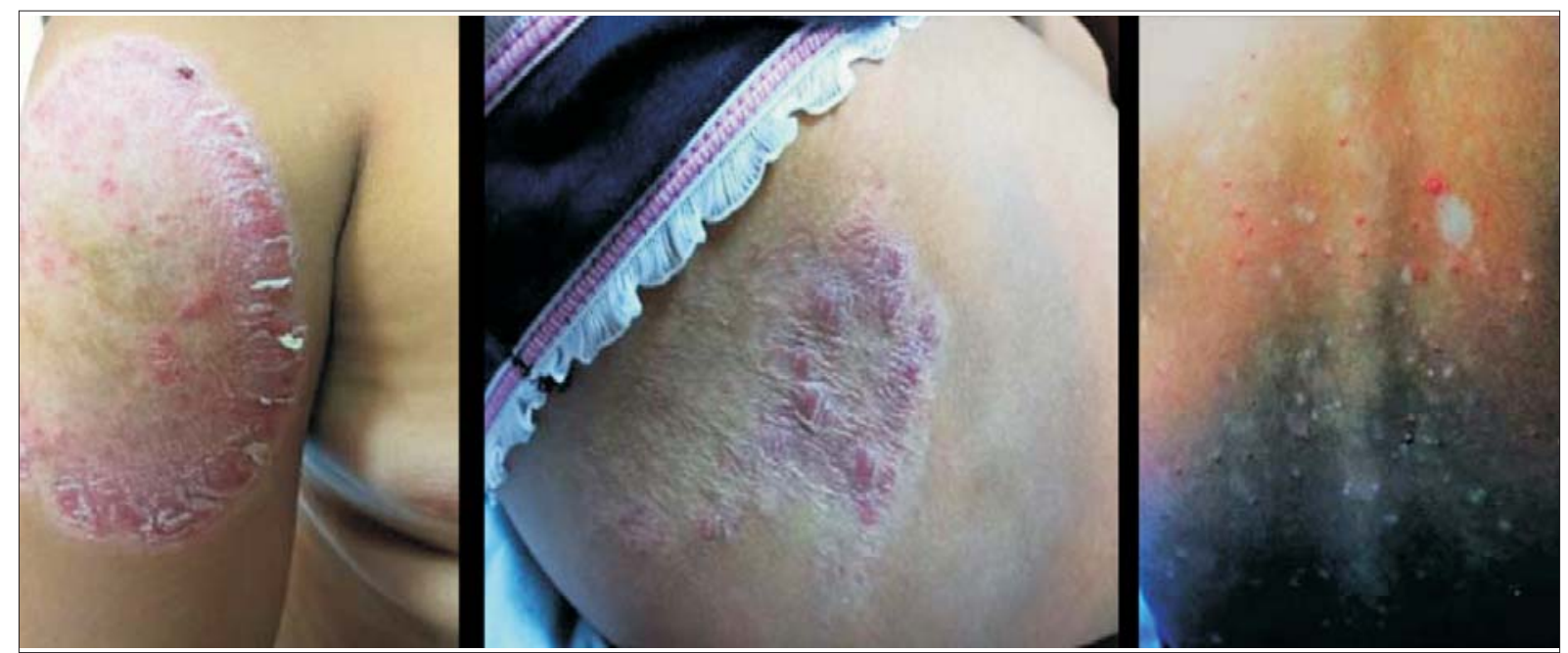

Figura 2. Aspecto de las lesiones en deltoides, muslo derecho y dorso al momento de la consulta.

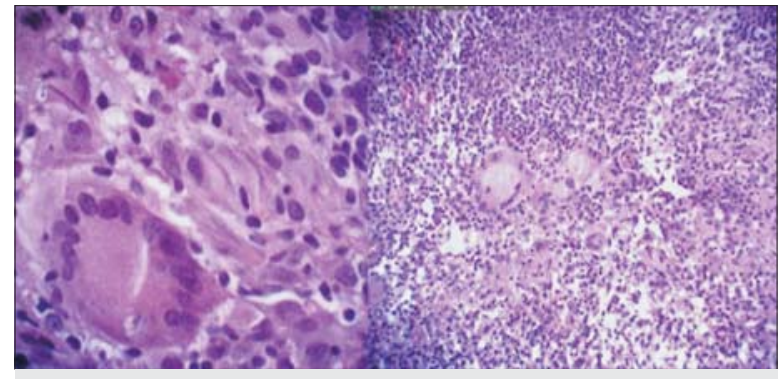

Figura 3. Anatomía patológica: aspecto histológico realizado con hematoxilina-eosina en biopsia de la lesión de brazo y muslo donde se observan los granulomas con necrosis caseosa central sugestivos de dermatitis tuberculosa.

Mycobacterium tuberculosis es el agente más común, pero también puede estar implicado Mycobacterium bovis y menos frecuentemente el $\mathrm{BCG}^{(11)}$. Esta forma de tuberculosis cutánea predomina en el sexo femenino en una relación 2:1 y se presenta en pacientes con moderado o alto grado de inmunidad como resultado de la diseminación de la micobacteria ya sea por vía hematógena, linfática o por contigüidad ${ }^{(4)}$. Raramente las lesiones se desarrollan sobre el punto de inoculación primaria o por vacunación $^{(3)}$. Se manifiesta como una o más placas asintomáticas eritemo parduzca de consistencia blanda, que a la discopía muestra el color típico de la jalea de manzana y donde existen micropápulas que se agrupan y coalesen (lupomas o tubérculos) formando un borde sobreelevado y una atrofia central debido a su crecimiento centrífugo ${ }^{(11-13)}$. Las lesiones provocadas por diseminación hematógena se localizan frecuentemente en cabeza y cuello, pero pueden afectar miembros y las mucosas

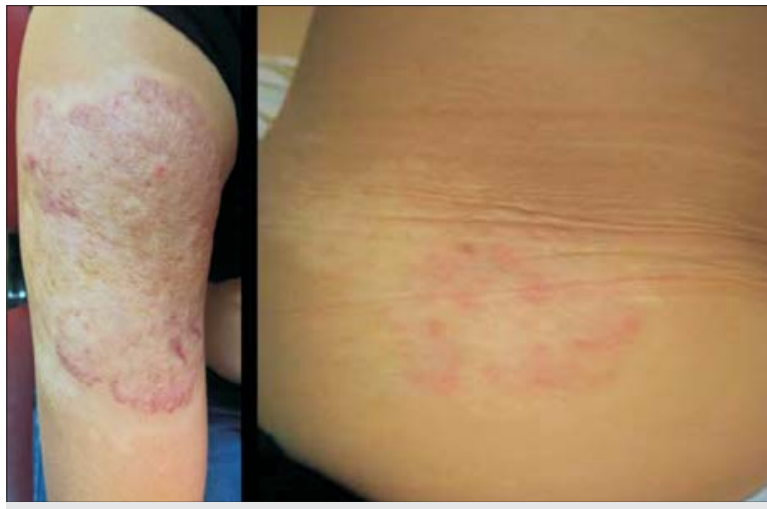

Figura 4. Aspecto de las lesiones del deltoides y muslo derecho luego de seis meses de tratamiento.

oral, nasal o conjuntival por extensión ${ }^{(14)}$. Las de reinoculación se localizan frecuentemente en los miembros. Son habitualmente lesiones únicas, pero se han descrito también formas múltiples ${ }^{(4,15)}$.

De las siete formas que se describen de LV, la paciente presentaba en los miembros superior e inferior derechos el tipo en placa que es el más fácil de diagnosticar, a pesar de esto no se pensó en esta entidad. Ya que la paciente presentó una lesión abscedada en la axila tras la inoculación con el BCG, pensamos que podría haberse tratado de otras formas de tuberculosis cutáneas que pueden asociarse a LV. La tuberculosis por inoculación primaria o chancro se caracteriza por una úlcera granulomatosa indolora con adenopatía regional que evoluciona hacia la supuración y se produce por inoculación externa o por el BCG. Las lesiones evolucionan a la curación espontánea con cicatrización en dos a 12 meses. 
La escrófula es otro proceso subcutáneo resultado de una diseminación por contigüidad desde un foco interno, ya sea ganglionar u óseo a la piel. Se localizan en regiones cervicales, axilares e inguinales. Es habitual que las lesiones terminen por fistulizarse y supurar. Puede curar espontáneamente luego de años y formarse una cicatriz de aspecto queloide.

Se ha descrito que en raras ocasiones se pueden producir lesiones de LV en cicatrices residuales de escrófulas o sobre o cercano al punto de inoculación de $\mathrm{BCG}^{(4,11,16)}$. Suponemos que fue lo que pudo haber ocurrido en esta niña luego de la vacunación, dado que el bacilo puede mantenerse latente muchos años en la lesión inicial y reactivarse, manifestándose luego como LV.

La paciente presentaba, además, lesiones múltiples en el dorso que hicieron sospechar en otras lesiones denominadas tubercúlides. Esta erupción cutánea, que hasta no hace mucho tiempo se pensaba que era producida por hipersensibilidad a M. tuberculosis o sus toxinas, hoy día se postula que probablemente se deba a diseminación hematógena en pacientes con enfermedad tuberculosa activa. Esta forma se ha visto que predomina en niños y adolescentes que han tenido el complejo primario, pero también se ha visto luego de la BCG y asociado a $L V^{(4,17)}$, situación que pensamos pudo haber ocurrido en esta paciente.

Se describe que una simple dosis de BCG puede causar LV, pero la incidencia de esta forma de tuberculosis aumenta con múltiples inyecciones. El tiempo entre la vacuna y la lesión de piel puede ser de varios meses a años, con una media de un año. Los factores que se describen como responsables y que inciden en esa evolución son la susceptibilidad del huésped, la virulencia del bacilo, la cantidad del inóculo y la técnica de inoculación ${ }^{(7)}$.

La vacunación con BCG sigue siendo el mejor método para prevenir las formas severas de enfermedad por M. tuberculosis. Más de 100 países continúan la vacunación en un esfuerzo por controlar la enfermedad. El 99\% de las vacunas son producidas de cinco diferentes cepas de M. bovis: la cepa Pasteur 1173P2, la danesa 1331, la Glaxo 1977 (derivada de la danesa), la Tokio 172-1, la BCG-1 rusa y la cepa brasileña Moreau RDJ. Las dos primeras son consideradas cepas fuertes, mientras que las últimas son consideradas débiles. Todas difieren en su inmunogenicidad, eficacia y efectos adversos. Han existido brotes de reacciones a BCG debido a cambios en los programas con las cepas a utilizar y dosis administradas. La respuesta usual es un área indurada de 5 a 15 mm que se reblandece en su centro por cuatro semanas y se forma una éscara que dura de seis a diez semanas, quedando luego una cicatriz o una adenitis menor a $1 \mathrm{~cm}^{(10,18-20)}$.
Desde su aplicación, hace ya 96 años, se han descrito complicaciones locales en 5\% de los vacunados, como formación de úlceras, linfadenitis supurativa, abscesos y reacciones queloides. Estas ocurren desde las dos semanas de la vacunación hasta tres meses después ${ }^{(4,18)}$. Se han descrito recientemente otras complicaciones, como son la formación de granulomas, LV, masas en la pared del tórax, eritrodermia con múltiples abscesos de piel, ulceraciones en el sitio de inoculación en el curso de la enfermedad de Kawasaki, etcétera ${ }^{(21,22)}$. Estas reacciones locales y regionales son en su mayoría autolimitadas. De las complicaciones sistémicas, la osteítis y la infección diseminada son más raras de observar. Ocurren en niños que presentan alguna IDP y ocurre dentro de los seis primeros meses de aplicada. La incidencia de enfermedad diseminada notificada por BCG es de 0,19 a 1,56 casos por millón de niños vacunados y las tasas de mortalidad oscilan entre $50 \%$ y $70 \%$.

Desde hace más de 20 años, en nuestro país, el Sistema Nacional de Farmacovigilancia y Efectos Supuestamente Atribuibles a la Vacunación e Inmunización del Ministerio de Salud es el lugar donde se deben denunciar todos estos eventos. En este caso en particular se investigó y no se encontraron datos de tal hecho.

Es importante destacar que en los casos en que suceden complicaciones locales o sistémicas se debe sospechar la presencia de una inmunodeficiencia, principalmente porque existe un grupo reducido de niños aparentemente sanos que presentan una IDP caracterizada por una susceptibilidad a enfermedades producidas por micobacterias (SMEM). Esta incluso se puede manifestar solamente por la presencia de una adenitis $\operatorname{axilar}^{(8,23)}$. Dado el carácter múltiple de las lesiones de la paciente, lo cual manifiesta una enfermedad diseminada por BCG y los antecedentes familiares, se sospechó que podría tener ese tipo de IDP. La SMEM se caracteriza por alteraciones del eje IL-12/IFN gama y dado que el estudio de rutina de estos pacientes suele ser normal, el diagnóstico de dicha IDP requiere comprobar la indemnidad del circuito SMEM. La deficiencia del receptor b-1 de la IL 12 es el defecto más común. Esta alteración puede ser parcial o total, por lo tanto la gravedad del fenotipo clínico dependerá del genotipo en cuestión. Lamentablemente no se pudo avanzar más en este diagnóstico debido a que los padres de la niña no asistieron más a los controles. Se ha descrito que aproximadamente la mitad de los pacientes con enfermedad por BCG, en los que no se encontró una IDP o ID secundaria, pueden tener un defecto del eje heredado. Las formas familiares y la consanguinidad de los padres son frecuentes y apoyarían el origen genético de esta alteración inmunológica.

Para el diagnóstico de la tuberculosis cutánea se siguen aplicando los criterios de Wilkinson modificados. 
En el caso de LV, la bacteria parece exhibir una baja virulencia y eso explicaría en parte lo difícil de evidenciarla por los métodos convencionales. Como criterio absoluto, el gold standar es el cultivo, mientras que los criterios relativos son los hallazgos clínicos, la histología, la reacción positiva a la tuberculina y la respuesta al tratamiento antituberculoso. La paciente presentaba un PPD reactivo, lo cual es habitual de observar en estos casos y solo demuestra el contacto previo con M. tuberculosis. La característica histológica típica es el granuloma tuberculoide, altamente sugestivo de la tuberculosis, pero que no es patognomónico ${ }^{(5)}$. En esta paciente habían sido objetivados los granulomas en la primera biopsia de piel. El no haber observado BAAR por la técnica de Zielh Nielsen, es habitual en la forma de LV, como también puede ocurrir que no haya necrosis caseosa. Si bien existen métodos de cultivo rápido, estos tampoco identifican el tipo de M. tuberculosis, que sí pueden resolver las técnicas de amplificación de ADN, como fue el caso de la paciente. Es fundamental en estos casos determinar la vinculación de la lesión a la vacuna, ya que $M$. bovis es resistente intrínsecamente a la pirazinamida y puede presentar una resistencia intermedia a la isoniacida ${ }^{(18)}$. Hay pocas guías de diagnóstico y tratamiento para enfermedad por BCG. En muchos laboratorios tampoco se estudia la susceptibilidad a los fármacos antituberculosos. De todas formas, se recomienda realizar la sensibilidad de dicho germen dado que se han descritos casos de enfermedad diseminada resistente a múltiples fármacos con desenlace fatal o crónico ${ }^{(8)}$.

El tratamiento se basa en el clásico esquema diario por dos meses y bisemanal por cuatro meses. Sin embargo, este va a depender del tipo de compromiso cutáneo, del nivel de inmunidad y de la gravedad del paciente. $\mathrm{Pa}$ ra el correcto manejo de estos pacientes, con infección regional o diseminada por BCG, es esencial asegurar la confirmación etiológica, usar dosis altas de fármacos antituberculosos y de forma prolongada. Hesselling y colaboradores, en un estudio realizado en el año 2006, donde evaluaron a niños con o sin VIH que presentaron complicaciones con la vacuna BCG, concluyen que no hay datos para recomendar un tratamiento anti-TB en niños inmunocompetentes con complicaciones locales a la vacuna. Sin embargo, en niños con VIH o con IDP utilizan cuatro fármacos a altas dosis por nueve a diez me$\operatorname{ses}^{(8,19)}$. Dada la alta sospecha de una IDP en la paciente, se decidió realizar un tratamiento más prolongado.

\section{Conclusión}

La tuberculosis cutánea ofrece un reto diagnóstico por ser infrecuente, por sus manifestaciones clínicas diversas y porque la identificación del bacilo en las lesiones es inusual.
Por otro lado, las complicaciones por BCG de por sí son muy raras. El LV puede aparecer en el lugar de la vacuna o cercana a ella hasta un año después de producida la inoculación. Puede persistir por décadas, reactivarse en sitios distantes, así como asociarse a otras lesiones de piel y dejar secuelas. Su diagnóstico debe ser considerado tanto en pacientes inmunocompetentes como inmunodeprimidos que presenten lesiones de evolución tórpida que no responden a los tratamientos instituidos. Se debe sospechar siempre una ID en niños que presentan complicaciones por la vacuna.

\section{Agradecimientos}

Al Dr. Fabricio Martínez, médico neumólogo de la CHLA, por su apoyo durante el tratamiento y seguimiento de la paciente.

\section{Abstract}

Cutaneous TB is a rather unusual chronic infectious disease caused by Mycobacteriun tuberculosis. There are several forms of cutaneous infection, lupus vulgaris being the most frequent and ordinary one in developed countries. Clinical variations will depend on the site of infiltration of the skin by the bacillus, the patient's immune condition and the environment. In the case of lupus vulgaris, apart from Mycobacteriun tuberculosis, $M$. bovis may be involved, and the Bacille Calmette Guérin involvement is less frequent. The latter presents a chronic evolution, being progressive and unusual in children.

The study presents the case of a girl who was diagnosed with a form of cutaneous tuberculosis called lupus vulgaris caused by the Bacille Calmette-Guérin vaccine.

Clinical case: a 10 year old girl who evidenced skin lesions of different kinds, of slow evolution and asymptomatic: the largest one was in the external part of the right deltoid muscle as an erythematous plaque of 20 by $10 \mathrm{~cm}$ with higher borders evidencing micro papules in the center that coalesce leaving a central atrophy zone. The latter had started in the neonatal stage after the Bacille Calmette-Guérin vaccine. For three years she had evidenced a second smaller lesion in the outer face of the right thigh of similar characteristics and other multiple lesions in the back with the shape of vinous red micro papules. Assessment tests were generally normal, as well as the chest and limbs X-ray. The tuberculin test evidenced a $15 \mathrm{~mm}$ induration and smears were negative. The deltoid muscle skin lesion biopsy showed caseous granulomas and molecular techniques diagnosed attenuated Mycobacteriumbovis. Given that diagnose the patient received anti-TB treatment during 10 months, tolerance being good and the lesions improved. 


\section{Resumo}

A tuberculose cutânea é uma doença infecciosa crônica e pouco frequente causada pelo Mycobacteriun tuberculosis. Existem várias formas de infecção cutânea, sendo o lúpus vulgar a mais frequente e comum nos países industrializados. As variantes clínicas dependerão da via de entrada do bacilo à pele, do estado imunológico do paciente e do ambiente. No lúpus vulgar, além do M. tuberculosis podem estar implicados o M. bovis e mais raramente o bacilo de Calmette-Guérin. Esta entidade apresenta uma evolução crônica, é progressiva e é rara em crianças.

Apresenta-se o caso de uma paciente de sexo feminino com 10 anos que foi diagnosticada com uma forma de tuberculose cutânea denominada lúpus vulgar provocada pelo bacilo de Calmette-Guérin.

Caso clínico: paciente de sexo feminino com 10 anos que apresentava lesões cutâneas de diferentes características, com evolução letárgica e assintomática; a maior lesão estava localizada na face externa do deltoide direito sob a forma de placa eritematosa de $20 \times 10 \mathrm{~cm}$ com bordes sobrelevados apresentando no centro micropápulas que coalescem deixando una zona de atrofia central. Esta lesão começou na etapa neonatal depois da vacinação com o bacilo de Calmette-Guérin. Desde 3 anos apresentava uma segunda lesão menor na face externa da coxa direita com características similares e outras múltiplas no dorso com forma de micropápulas com coloração de manchas em vinho do Porto. Os exames de avaliação geral foram normais, bem como as radiografias de tórax e de membros. O teste da tuberculina mostrou uma induración de $15 \mathrm{~mm}$ e as baciloscopias foram negativas. A biopsia da lesão de pele dos deltoides mostrou granulomas caseosos e as técnicas moleculares diagnosticaram Mycobacterium bovis atenuado. Com esse diagnóstico recebeu tratamento antituberculoso por 10 meses com boa tolerância e melhoria das lesões.

\section{Bibliografía}

1. Bisero E, Luque G, Melillo K, Favier M, Zapata A, Cuello M. Tuberculosis cutánea: reporte de un caso. Arch Argent Pediatr 2014; 112(3):e93-6.

2. Jaka-Moreno A, López-Nuñez M, López-Pestaña A, Tuneu-Valls A. Lupus vulgar causado por Mycobacterium bovis. Actas Dermosifiliogr 2012; 103(3):251-3.

3. Panzarelli A. Tuberculosis cutánea. Derm Venez 1988; 36(2):45-51.

4. Tincopa Wong O, Sanchez-Saldaña L. Tuberculosis cutánea. Dermat peru 2003; 13(3):195-214.

5. Almaguer-Chávez J, Ocampo-Candiani J, Rendón A. Panorama actual en el diagnóstico de la tuberculosis cutánea. Actas Dermosifiliogr 2009; 100:562-70.
6. Contrera M. Situación epidemiológica de la tuberculosis en Uruguay. En: CHLA-EP, MSP, UdelaR-Facultad de Medicina-Cátedras de Enfermedades Infecciosas y de Neumología. Guía Nacional para el Manejo de la Tuberculosis. $3^{\mathrm{a}}$ ed. Montevideo: CHLA-EP, 2016:17-21.

7. Afsar FS, Afsar I, Diniz G, Asilsoy S, Sorguc Y. Lupus vulgaris in a pediatric patient: a clinicohistopathological diagnosis. Braz J Infec Dis 2008; 12(2):152-4.

8. Najem NM, Zadeh VB, Al-Abdulrazzaq AH, Al-Otaibi SR, Kadyan S, Joneja M. Bacillus Calmette-Guérin vaccine induced lupus vulgaris in a child. Acta Dermatovenerol Alp Pannonica Adriat 2009; 18(4):195-7.

9. Strickler A, Pérez A, Risco M, Gallo S. Enfermedad por bacilo de Calmette-Guérin (BCG) y deficiencia del receptor b-1 de interleuquina 12: experiencia clínica de dos casos en una familia y un caso aislado. Rev chil infectol 2014; 31(4):444-51.

10. Venkataraman A, Yussuff M, Liebeschuetz S, Riddell A, Prendergast AJ. Management and outcome of Bacille Calmette-Guérin vaccine adverse reactions. Vaccine 2015; 33:5470-4.

11. Spelta K, Diniz L. Cutaneous tuberculosis: a 26-year restrospective study in a endemic área of tuberculosis, Vitória, Espírito Santo, Brazil. Rev Inst Med Trop Sao Paulo 2016; $58: 49$.

12. Motta A, Feliciani C, Toto P, De Benedetto A, Morelli F, Tulli A. Lupus vulgaris developing at the site of misdiagnosed scrofuloderma. J Eur Acad Dermatol Venereol 2003; 17(3):313-5.

13. Yanine D, Latorres M, Campos F, Orozco C. Tuberculosis cutánea: revisión del tema a propósito de dos casos. Rev Chilena Dermatol 2015; 31(2):155-60.

14. Gómez Sierra M, Bolomo G, Ibañez M, Miraglia E, Campoy $\mathbf{V}$, Palazzolo J, et al. Tuberculosis cutánea: lupus vulgar. Arch Argent Dermatol 2016; 66(3):82-5.

15. izzariello G, Fernández Pardal P, D`Atri G, Novac V, Uranga A. Espectro clínico de la tuberculosis cutánea. Rev Argent Dermatol 2008; 89:177-87.

16. Bhardwaj P, Mahajan V. Lupus vulgaris. Indian Pediatr 2003; 40:902-3.

17. Meltem Akkurt Z, Uçmak D, Fidan V, Türkcü G. Tuberculide papulonecrótica. Caso clínico. Arch Argent Pediatr 2013; 111(1):e5-7.

18. Concha M, Fich F, Rabahagliati R, Pinto C, Rubio R, Rocío, Navea O. Tuberculosis cutánea: reporte de dos casos y revisión de la literatura. Rev Chil Infectol 2011; 28(3):262-8.

19. Lussier N, Bourgault A, Gaundreau C, Turgeon P. A complication of BCG vaccine: a case of localized cutaeous abscess due to Mycobacterium bovis. Can J Infect Dis 1999; 10(3):257-9.

20. Hesseling A, Rabie H, Marais J, Manders M, Lips M, Schaaf S, et al. Bacille Calmette-Guérin vaccine-induced disease in HIV-infected and HIV-uninfected children. Clin Infect Dis 2006; 42:548-58.

21. Organización Mundial de la Salud. Hoja de información sobre las tasas observadas de reacciones a vacunas: Vacuna 
bacilo de Calmette-Guérin (BCG). Ginebra: OMS, 2012. Disponible en: http://www.who.int/vaccine_safety/initiative/tools/BCG_Vaccine_rates_information_sheet_ES.pdf [Consulta: 26 marzo 2018]

22. Bellet JS, Prose NS. Skin complications of Bacillus Calmette-Guérin inmunization. Curr Opin Infect Dis.2005; 18(2): 97-100.
23. Santos A, Dias A, Cordeiro A, Cordinhã C, Lemos S, Rocha G, et al. Severe axillary limphadenitis after BCG vaccination: alert for primary immunodeficiencies. J Microbiolol Inmunol Infect 2010; 43(6):530-7. 